\title{
Multiple Pulmonary Nodules in Ectopic Adrenocorticotropic Hormone Syndrome: Cause or Result?
}

\author{
Shan-Yueh Chang a Tzu-Chuan Huang ${ }^{b}$ Wen-Lin Su ${ }^{a}$ Chih-Feng Chian ${ }^{a}$ \\ Wann-Cherng Perng ${ }^{a}$ \\ Divisions of a Pulmonary and Critical Care Medicine and ${ }^{b}$ Hematology/Oncology, Department of Internal Medicine, \\ Tri-Service General Hospital, Taipei, Taiwan
}

\section{Key Words}

Ectopic adrenocorticotropic hormone syndrome •

Pulmonary nodules $\cdot$ Opportunistic infections

\begin{abstract}
Objective: To report a case of invasive pulmonary aspergillosis mimicking lung cancer with lung to lung metastases in ectopic adrenocorticotropic hormone syndrome (EAS). Clinical Presentation and Intervention: A 60-year-old man suffering from hypokalemic alkalosis, hypertension and limbs paralysis was referred to our hospital. EAS caused by malignancy of lung was highly suspected due to multiple pulmonary nodules presenting on chest film and positron emission tomography (PET) images. Video-assisted thoracic surgical biopsy tissue was used to confirm invasive aspergillosis instead of malignancy. Finally, the patient died of opportunistic infection. Conclusion: This case showed that although EAS is usually associated with solid tumors, multiple pulmonary nodules secondary to opportunistic infections such as invasive aspergillosis must be kept in mind.
\end{abstract}

Copyright $\odot 2012$ S. Karger AG, Basel
(C) 2012 S. Karger AG, Basel

1011-7571/12/0213-0292\$38.00/0

Accessible online at:

www.karger.com/mpp

\section{Introduction}

Ectopic adrenocorticotropic hormone syndrome (EAS) is a well-known paraneoplastic disorder accounting for $12-17 \%$ of cases of Cushing's syndrome and is associated with a variety of solid tumors, mostly differentiated neuroendocrine tumors including small cell lung cancer [1]. The classic cushinoid appearance, such as striae, moon face and truncal obesity may be absent or minimal in patients with EAS, possibly due to the aggressive clinical course of underlying malignancy. Hypertension is common (62-92\%), as well as hypokalemia (71-100\%) and metabolic alkalosis (more than 80\%) [2]. The diagnostic steps are complicated, including clinical features, biochemical abnormalities, plasma adrenocorticotropic hormone (ACTH), cortisol, urinary steroids, high-dose dexamethasone suppression test, inferior petrosal sinus ACTH sampling and imaging studies. However, the primary etiology was not detected in $12 \%$ of the cases [2]. Patients with hypercortisolism are especially at risk of opportunistic infections due to complex immunosuppressive mechanisms, such as impaired cellular immunity, decreased recruitment of macrophages and increasing host susceptibility to invasive fungal infection [3]. We 


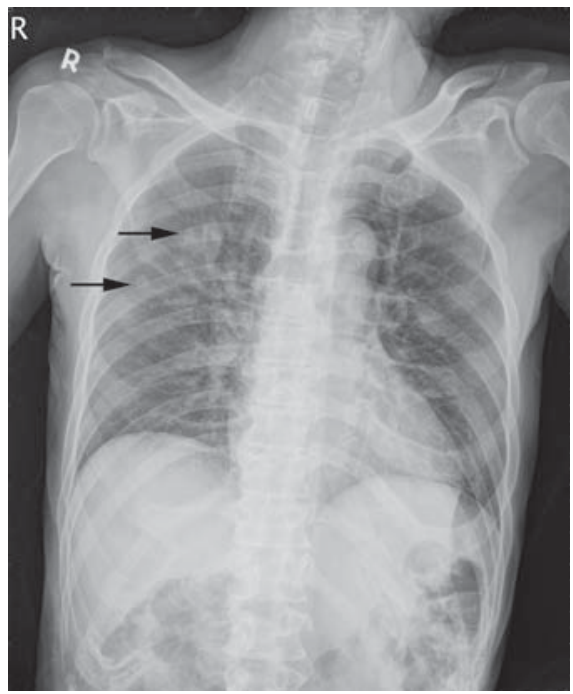

Fig. 1. Irregular nodular opacities over the right upper lobe of the lung (arrows).
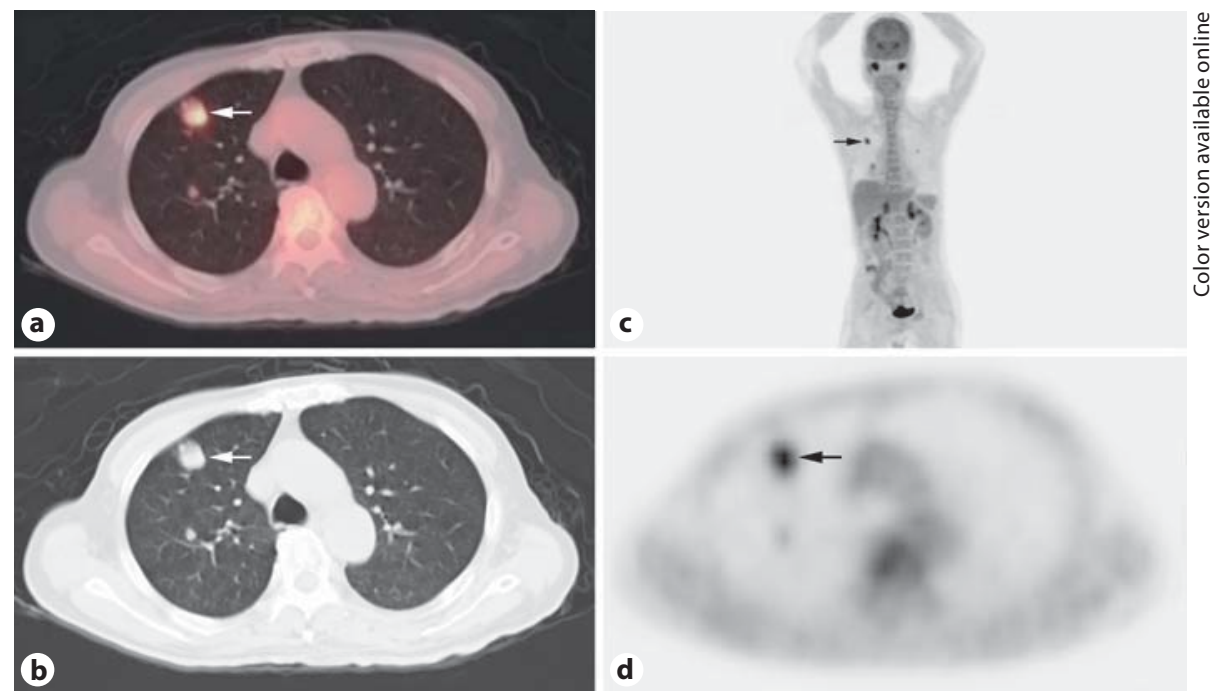

(b)

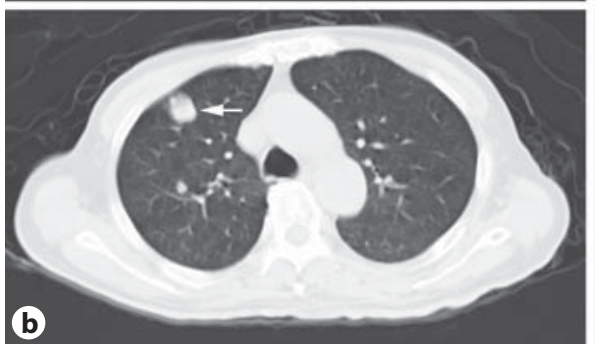

Fig. 2. a PET/CT. b CT. c Maximal intensity projection imaging. d PET. Arrows indicate a right upper lobe nodule with an increased SUV.

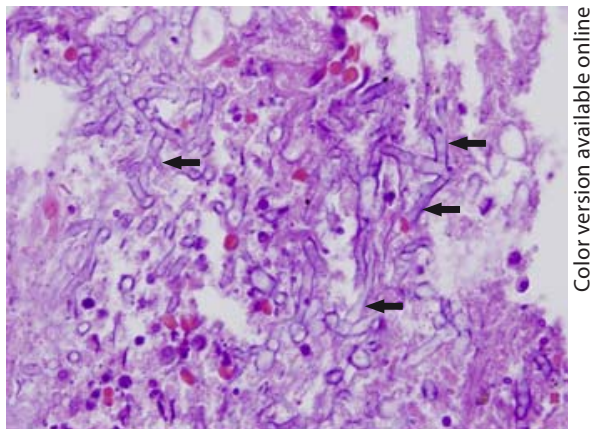

Fig. 3. Foci of necrotic tissue and abscess mixed with scattered foci of fungal colonies that are predominantly composed of Aspergillus-like hyphae (arrows). HE. Original magnification $\times 1,000$.

report a 60 -year-old patient who was misdiagnosed as having EAS caused by lung cancer because of multiple pulmonary nodules.

\section{Case Report}

A 60-year-old man was hospitalized due to progressive lower limbs paralysis and hypertension of one month duration. Based on his past medical history, no major systemic diseases or exposure of significant drug insult were noted. Biochemical studies revealed severe hypokalemia $(1.9 \mathrm{mEq} / \mathrm{l}$, range $3.5-5.1)$ and metabolic alkalosis. Normal serum aldosterone $(85.1 \mathrm{pg} / \mathrm{ml}$, range $35.7-240)$ and raised cortisol level $(158.2 \mu \mathrm{g} / \mathrm{dl}$ at 6 a.m., range
9-53) were also detected. Severe hypokalemia caused by Cushing's syndrome was first considered. His symptoms relieved gradually after potassium supplement. However, patients refused further interventions to clarify the etiology, and then he was discharged without the final diagnosis and with serum value of ACTH (425 pg/ml, 0.1-46). Because of high level of ACTH, EAS was suspected, hence magnetic resonance imaging (MRI) of brain was performed, but revealed no pituitary lesion. The patient was admitted again due to progressively general malaise and body weight loss from 63 to $58 \mathrm{~kg}$. A standing chest radiograph (fig. 1) revealed irregular patch opacities over the right upper lobe which were not visible 1 month ago. Bacterial cultures, sputum cytology, acid-fast stain of sputum and blood cryptococcal antigen test were all negative. Positron emission tomography/computerized tomography (PET/CT) was suggested and demonstrated multiple pulmonary nodules with the largest size of $1.6 \mathrm{~cm}$ and the maximal standardized uptake value (SUV ${ }_{\text {max }}$ ) of 3.7 (fig. 2). Lung cancer with lung-to-lung metastases was highly suspected. Bronchoscopy revealed no endobronchial lesion. Computer tomographicguided lung biopsy showed no malignant cells. Video-assisted thoracic surgery (VATS) of right upper lobe was performed, and Aspergillus-like hyphae were found (fig. 3). No malignancy was noted. Aspergillus spp. cultured from the lung tissue confirmed the final diagnosis of invasive pulmonary aspergillosis. The patient was transferred to intensive care unit due to hypoxemia and rapidly deteriorating disease. Broad-spectrum antibiotics and antifungal agent were administered. Acute respiratory distress syndrome developed three days after VATS, followed by septic shock with Pneumocystis jiroveci fungemia and Cytomegalovirus (CMV) viremia, which were confirmed by polymerase chain reaction (PCR) of blood sample via blood. Oral ketoconazole of $1,200 \mathrm{mg}$ once daily was prescribed because of the persistent hypercortisol level with life-threatening opportunistic infection. Multiple organ dysfunction syndrome developed afterwards, and the patient expired despite the medical intervention. 


\section{Discussion}

Plasma ACTH values of more than $200 \mathrm{pg} / \mathrm{ml}$ were reported in $33-72 \%$ of the EAS patients reviewed and had tended to be higher than those in Cushing's disease [2]. Nevertheless, there is still no clear cutoff value between EAS and Cushing's syndrome. In our patient, matched clinical manifestations, higher plasma ACTH value (425 $\mathrm{pg} / \mathrm{ml}$ ) and negative findings of brain MRI strongly supported the diagnosis of EAS, even though other confirmatory tests were not performed because of the rapid deterioration of the patient.

Therefore, it was reasonable to assume that the etiology was due to malignancy because of the evidences mentioned below. First, the largest pulmonary nodule was $1.6 \mathrm{~cm}$ in size in our case as well as high SUV value obtained in PET/CT scan. Second, approximately $45 \%$ of paraneoplastic EAS result from small cell lung cancer, $15 \%$ thymic carcinoid tumors, $10 \%$ bronchial carcinoid tumors, $10 \%$ pancreatic islet cell tumors, $5 \%$ other carcinoid and 2\% pheochromocytomas [4]. Third, multiple pulmonary nodules with sharply demarcated borders are often metastases, whereas primary lung cancer with lung metastases are often quite irregular [5]. Equally, invasive fungal infections are common in patients with ectopic ACTH production, probably due to the hypercortisol level causing impairment to cell-mediated immunity [6]. Although the typical finding of CT halo sign was not seen in our case, multiple areas of consolidation or small nodules could be due to invasive aspergillosis [7]. Therefore, CT-guided biopsy was done in our patient because it was important to clarify the etiology of pulmonary nodules seen on PET/CT images and to decide on optimum treatment modality. It should be pointed out that $75 \%$ of pulmonary complications in immunocompromised patients are due to infection, which is associated with high morbidity and mortality [8]. In EAS, the degree of elevated cortisol level directly influences the etiology of opportunistic infection, especially invasive aspergillosis, cryptococcosis and $P$. jiroveci pneumonia [3].

Treatment of EAS with opportunistic infection necessitates not only early administration of appropriate systemic antimicrobial therapy, but control of the predisposing hypercortisolism. According to previous review, 15 of the 25 described patients with hypercortisolism and opportunistic infections died of the infection [9]. Hence, control of hypercortisolism with adrenal enzyme inhibitors, glucocorticoid antagonist, and even medical adrenalectomy should be completed as soon as possible. Surgical adrenalectomy is also considered if life-threatening infection and refractory hypercortisolism persists despite conventional anticortisolic medications [10].

\section{Conclusion}

This case showed that although EAS is usually associated with solid tumors, multiple pulmonary nodules secondary to opportunistic infections such as invasive aspergillosis must be kept in mind. Invasive procedures should be initiated as early as possible to avoid delay in treatment since nodules may be composed of both tumor and infectious agents.

\section{References}

1 Isidori AM, Lenzi A: Ectopic ACTH syndrome. Arq Bras Endocrinol Metabol 2007; 51:1217-1225.

2 Wajchenberg BL, Mendoca BB, Liberman B, Pereira MAA, Kirschner MA: Ectopic ACTH syndrome. J Steroid Biochem Molec Biol 1995;53:139-151.

-3 Lionakis MS, Kontoyiannis DP: Glucocorticoids and invasive fungal infections. Lancet 2003;362:1828-1838.

-4 Salgado LR, Fragoso MC, Knoepfelmacher M, Machado MC, Domenice S, Pereira MA, Mendonca BB: Ectopic ACTH syndrome: our experience with 25 cases. Eur J Endocrinol 2006; 155:725-733.
5 Gross BH, Glazer GM, Bookstein FL: Multiple pulmonary nodules detected by computed tomography: diagnostic implications. J Comput Assist Tomogr 1985;9:880-885.

-6 Sutton BJ, Parks GE, Manavi CK, Palavecino EL, Geisinger KR: Cushing's syndrome and nocardiosis associated with a pulmonary carcinoid tumor: report of a case and review of the literature. Diagn Cytopathol 2011;39: 359-362.

7 Horger M, Hebart H, Einsele H, Lengerke C, Claussen CD, Vonthein R, Pfannenberg C: Initial CT manifestations of invasive pulmonary aspergillosis in 45 non-HIV immunocompromised patients: association with patient outcome? Eur J Radiol 2005;55:437444.
-8 Oh YW, Effmann EL, Godwin JD: Pulmonary infections in immunocompromised hosts: the importance of correlating the conventional radiologic appearance with the clinical setting. Radiology 2000;217:647-656.

-9 Sieber SC, Dandurand R, Gelfman N, Iannini P, Braza F: Three opportunistic infections associated with ectopic corticotropin syndrome. Arch Intern Med 1989;149:25892591.

10 Joubert M, Reznik Y, Verdon R: 'Rescue' bilateral adrenalectomy in paraneoplastic Cushing's syndrome with invasive Aspergillus fumigatus infection. Am J Med Sci 2007; 334:497-498. 